\title{
An Extension of the Generalized Hamiltonian Method to $S$-parameter Descriptor Systems
}

\author{
Zheng Zhang and Ngai Wong \\ Department of Electrical and Electronic Engineering, \\ The University of Hong Kong, \\ Pokfulam Road, Hong Kong. \\ Emails: \{zzhang,nwong\}@ eee.hku.hk
}

\begin{abstract}
A generalized Hamiltonian method (GHM) was recently proposed for the passivity test of hybrid descriptor systems [1]. This paper extends the GHM theory to its $S$-parameter counterpart. Based on the $S$-parameter GHM, a passivity test flow is proposed, which is capable of detecting nonpassive regions of descriptor-form physical models. The proposed method is applicable to $S$-parameter and hybrid systems either in the standard state-space or descriptor forms. Experimental results confirm the effectiveness and accuracy of the proposed method.
\end{abstract}

\section{INTRODUCTION}

In this paper we consider the linear time-invariant (LTI) descriptor system (DS) described by the state-space equations

$$
\begin{aligned}
& E \frac{d x}{d t}=A x+B u, \\
& y=C x+D u .
\end{aligned}
$$

Here $E, A \in \mathbb{R}^{n \times n}, B \in \mathbb{R}^{n \times m}, C \in \mathbb{R}^{m \times n}, D \in \mathbb{R}^{m \times m}$, $x \in \mathbb{R}^{n}$ represents state variables. In this DS, the matrix pencil $(A, E)$ is assumed to be regular, i.e., $\operatorname{det}(A-s E) \neq 0$ for some $s$ on the complex plane. Note that $\operatorname{rank}(E) \leq n$, and (1) describes a regular system if $E$ is invertible.

In circuit simulation, the original models obtained by modified nodal analysis (MNA) are in the descriptor form [2-4]. DSs also appear in full-wave simulations of 3-D structures, RF components and electromagnetic devices [5-7]. These original large-scale DS models are normally approximated by model order reduction (MOR) techniques to speed up computer-aided design (CAD) and simulations [2-8]. Meanwhile, compact models can also be built from measured frequency-dependent admittance, impedance or scattering response data by vector fitting [9]. In high-frequency simulations where the port excitations are wave-like, $S$-parameter representations are frequently adopted.

In VLSI CAD, these models are required to be passive to guarantee stable global simulations. Normally they are tested by passivity assessments and then processed by passivity enforcement techniques if necessary [10]. For standard state-space models, various passivity tests have been proposed, among which frequency sweeping and Hamiltonian method are most widely used $[10,11]$. Compared with sweeping routines, Hamiltonian method is frequency-independent and thus much more accurate [10]. Recently, a generalized Hamiltonian method (GHM) has been proposed for hybrid (i.e., impedance or admittance) DSs [1]. As a superset of the Hamiltonian method, GHM test enjoys much cheaper computations than existing algebraic DS passivity tests, e.g., linear matrix inequality (LMI) test and some decompose-and-test flows [12-14]. GHM test is also much more accurate than DS frequency sweeping method [15].

However, little work has been reported for the passivity test of $S$-parameter DSs. Although extended bounded-real lemma [16] and GARE-based method [4] have been proposed for passivity check of $S$-parameter DSs, no reliable technique exists for their passive/nonpassive region identification. This is what motivates this work. In this paper, we extend the GHM theory to $S$-parameter DS models. Analogous to its hybrid counterpart, the $S$-parameter GHM method can accurately identify the possible nonpassive frequency regions of both descriptor and regular models, without restrictions on the matrix $D$. Furthermore, we also show that our proposed method can test the passivity of hybrid DSs.

Throughout this paper, we use the superscripts ${ }^{T}$ and ${ }^{*}$ to denote transpose and conjugate transpose (Hermitian), respectively. For a general matrix $X, \sigma(X)$ represents the set of singular values of $X$. We use $\lambda(X)$ or $\lambda(X, Y)$ to denote the eigenvalues of $X$ or the generalized eigenvalues of the matrix pencil $(X, Y)$ (i.e., $\operatorname{det}(X-a Y)=0$ if $a \in \lambda(X, Y)$ ), respectively.

\section{TheOrETICAL BACKGROUND}

\section{A. Passivity Check of S-Parameter Models}

In the case of scattering representation, (strict) passivity is equivalent to the (strict) bounded realness of the transfer matrix $S(s)$. A transfer matrix $S(s) \in \mathbb{C}^{m \times m}$ is bounded real iff [17]:

1. $S(s)$ is analytical on the right half plane $\operatorname{Re}(s) \geq 0$;

2. $I-S^{*}(j \omega) S(j \omega) \geq 0$ (> corresponds to strict bounded realness) for all $\omega \in \mathbb{R}$, which is equivalent to

$$
\sigma_{i}(j \omega) \leq 1, \forall \sigma_{i}(j \omega) \in \sigma(S(j \omega)), \forall \omega \in \mathbb{R}
$$

Condition 2 can be checked by frequency sweeping techniques at a set of sampling points along the imaginary axis $s=j \omega[11,15]$. However, erroneous results may be produced because nonpassive regions between sampling points may be missed. For standard state-space models (with $E=I$ in (1)), 
the following $2 n \times 2 n$ Hamiltonian matrix

$$
\Pi_{S}=\left[\begin{array}{cc}
A-B D^{T} \hat{S}^{-1} C & -B \hat{R}^{-1} B^{T} \\
C^{T} \hat{S}^{-1} C & C^{T} D \hat{R}^{-1} B^{T}-A^{T}
\end{array}\right]
$$

is used for accurate passivity test [10]. Here $\hat{S}=\left(D D^{T}-I\right)$ and $\hat{R}=\left(D^{T} D-I\right)$. Any purely imaginary eigenvalue of $\Pi_{S}$ $j \hat{\omega}$ corresponds to a crossover frequency point $\hat{\omega}$ (in $\mathrm{rad} / \mathrm{sec}$ ) of passivity violations. Due to its frequency-independent nature, Hamiltonian method is much more accurate than frequency sweeping test and thus preferred in passivity enforcements where accurate passivity assessment is required. However, there still exist some problems for Hamiltonian method. Firstly, it is only applicable to standard state-space models. Secondly, as shown in (3), $I-D^{T} D$ is required to be nonsingular for passivity test.

\section{B. GHM Test for Hybrid Descriptor Systems}

Ref. [1] has proposed the GHM for DSs with admittance/impedance transfer matrix. In GHM test, the possible impulsive part is tested in advance by a preprocess called ImPT without system decomposition [1]. The proper part is checked by a matrix pencil $(J, K)$ :

$$
\begin{aligned}
& J=\left[\begin{array}{cc}
A-B\left(D+D^{T}\right)^{-1} C & -B\left(D+D^{T}\right)^{-1} B^{T} \\
C^{T}\left(D+D^{T}\right)^{-1} C & C^{T}\left(D+D^{T}\right)^{-1} B^{T}-A^{T}
\end{array}\right] \\
& K=\left[\begin{array}{rr}
E & \\
& E^{T}
\end{array}\right] .
\end{aligned}
$$

Since every purely imaginary generalized eigenvalue of $(J, K)$ corresponds to a crossover point, system passivity can be easily checked and the possible nonpassive regions can be accurately located. The main computation of GHM test is the $O\left(n^{3}\right)$ generalized eigenvalue solution. Due to its flexibility, high accuracy and explicit implementation, GHM method is generally superior over previous DS passivity tests [1].

\section{GHM THEORY FOR $S$-PARAMETER DSS}

Since the passivity of $S$-parameter models are characterized by $\sigma(S(j \omega))$, we consider the connections of the operation frequency with the singular values of $S(j \omega)$.

Theorem: For the DS defined in (1) with $j \omega \notin \lambda(A, E)$ and $\gamma \notin \sigma(D)$, we have $\gamma \in \sigma(S(j \omega))$ iff $j \omega \in \lambda(M, N)$ with

$$
\begin{aligned}
& M=\left[\begin{array}{cc}
A-B D^{T} S^{-1} C & -\gamma B R^{-1} B^{T} \\
\gamma C^{T} S^{-1} C & -A^{T}+C^{T} D R^{-1} B^{T}
\end{array}\right] \\
& N=\left[\begin{array}{cc}
E & \\
& E^{T}
\end{array}\right],
\end{aligned}
$$

where $S=D D^{T}-\gamma^{2} I, R=D^{T} D-\gamma^{2} I$. Since $D^{T} S^{-1}=$ $\left(R^{T}\right)^{-1} D^{T}, M$ is a Hamiltonian matrix.

With the assumption of $(A, E)$ being regular, the $S$ parameter transfer matrix of the DS in (1) can be written as

$$
S(j \omega)=C(j \omega E-A)^{-1} B+D .
$$

For real matrix-valued systems we have $S^{*}(j \omega)=S^{T}(-j \omega)$, therefore,

$$
S(j \omega)=B^{T}\left(j \omega E^{T}+A^{T}\right)^{-1}\left(-C^{T}\right)+D^{T} .
$$

Assume $\gamma \in \sigma(S(j \omega))$ and $\gamma \notin \sigma(D)$, then there exist non-zero $v$ and $u$ as the corresponding left-singular and rightsingular vectors, respectively, such that

$$
S(j \omega) u=\gamma v, \quad S^{*}(j \omega) v=\gamma u .
$$

Substituting (6) and (7) into the above equation, we rewrite (8) as

$$
\begin{aligned}
& {\left[\begin{array}{ll}
C & \\
& B^{T}
\end{array}\right] \Omega_{\omega}^{-1}\left[\begin{array}{ll}
B & \\
& -C^{T}
\end{array}\right]\left[\begin{array}{l}
u \\
v
\end{array}\right]} \\
& =\left[\begin{array}{cc}
-D & \gamma I \\
\gamma I & -D^{T}
\end{array}\right]\left[\begin{array}{l}
u \\
v
\end{array}\right],
\end{aligned}
$$

where

$$
\Omega_{\omega}=\left[\begin{array}{ll}
j \omega E-A & \\
& j \omega E^{T}+A^{T}
\end{array}\right] .
$$

For simplicity, we further denote

$$
z:=\Omega_{\omega}^{-1}\left[\begin{array}{ll}
B & \\
& -C^{T}
\end{array}\right]\left[\begin{array}{l}
u \\
v
\end{array}\right] .
$$

Because the right-hand side of (9) is non-zero, $z$ should also be a non-zero vector. From (9) and (11), it is straightforward to get

$\Omega_{\omega}^{-1}\left[\begin{array}{cc}B & \\ & -C^{T}\end{array}\right]\left[\begin{array}{cc}-D & \gamma I \\ \gamma I & -D^{T}\end{array}\right]^{-1}\left[\begin{array}{ll}C & \\ & B^{T}\end{array}\right] z=z$.

Pre-multiplying both sides of (12) by $\Omega_{\omega}$ yields

$$
M z=j \omega N z
$$

The converse also holds. To prove this, we first define

$$
z^{\prime}:=\left[\begin{array}{cc}
-D & \gamma I \\
\gamma I & -D^{T}
\end{array}\right]^{-1}\left[\begin{array}{ll}
C & \\
& B^{T}
\end{array}\right] z
$$

and then we can return to (9) and (8) via multiplying by $\left[\begin{array}{cc}-D & \gamma I \\ \gamma I & -D^{T}\end{array}\right]^{-1}\left[\begin{array}{ll}C & \\ & B^{T}\end{array}\right]$ on both sides of (12).

To this end, we have extended the GHM theory to $S$ parameter DSs.

\section{Passivity Test of DSs}

The passivity of scattering DSs are assessed by the bounded realness of its transfer matrix. For hybrid DSs, the passivity is tested based on the positive realness conditions. In this part, we show the application of $S$-parameter GHM in passivity test of both scattering and hybrid DSs.

\section{A. Passivity Test of S-Parameter DSs}

The GHM theory proposed in Section III has revealed a close relationship between the singular value $\gamma$ of a DS transfer matrix and its operation frequency $\omega$. We are interested in the case 
of $\gamma=1$, which represents the boundary of passivity violations of a $S$-parameter model.

Set $\gamma=1$, then we get a passivity test matrix pencil $(M, N)=\left(M_{0}, N_{0}\right)$ for scattering DSs with

$$
M_{0}=\Pi_{S}, \quad N_{0}=N .
$$

Here $\Pi_{S}$ is the Hamiltonian matrix defined in (3). If the matrix pencil $\left(M_{0}, N_{0}\right)$ has a purely imaginary generalized eigenvalue $j \hat{\omega}$, then $\hat{\omega}$ is a crossover frequency point where passivity violation happens. Therefore, via the generalized eigenvalue solution of the above matrix pencil, the possible nonpassive regions can be accurately located.

When $E$ is invertible, the DS in (1) reduces to a regular system which can be converted to a standard state-space model with $E=I$. In such case, the generalized eigenvalue solution in the test pencil of $\left(M_{0}, N_{0}\right)$ can be replaced by the standard eigenvalue problem of $M_{0}=\Pi_{S}$. This is in fact the traditional Hamiltonian method in (3), which is widely applied in passivity test and compensations of standard state-space models (see [10] and references therein). Therefore, the Hamiltonian method in (3) is a special case (or subset) of the proposed GHM theory.

\section{B. Passivity Test of Hybrid DSs}

A LTI system with hybrid (admittance or impedance) transfer matrix is (strictly) passive if and only if its transfer matrix being (strictly) positive real. Given transfer matrix $H(s) \in$ $\mathbb{C}^{m \times m}$, it is positive real if and only if [12]:

1. $H(s)$ has no poles in $\operatorname{Re}(s)>0$;

2. $H(s)$ is real for all positive real $s$;

3. $H(s)+H^{*}(s) \geq 0$ for all $\operatorname{Re}(s)>0$.

Given a square transfer matrix $H(s)$ with $\operatorname{det}(I+H(s)) \neq 0$ for all $\operatorname{Re}(s)>0$, a Moebius transformation of $H(s)$ is defined as [4]

$$
G(s)=(I-H(s))(I+H(s))^{-1} .
$$

The system matrices of $G(s)$ can be constructed as

$$
\begin{aligned}
& E_{S}=E, A_{S}=A-B(I+D)^{-1} C, \\
& B_{S}=-\sqrt{2} B(I+D)^{-1}, C_{S}=\sqrt{2}(I+D)^{-1} C, \\
& D_{S}=(I-D)(I+D)^{-1} .
\end{aligned}
$$

Since $D$ usually has a much lower dimension than $E$ and $A$, the above transformation is of very low computational cost. It is worth noting that $H(s)$ itself is also a Moebius-transformed transfer function of $G(s)$.

An important property of Moebius transformation is that $H(s)$ is positive real if and only if its Moebius-transformed function $G(s)$ is bounded real [4]. If we see $G(s)$ as a scattering system, then $H(s)$ is passive if and only if $G(s)$ is passive. Therefore, the passivity of $H(s)$ can be tested via the $S$-parameter GHM test on $G(s)$.

\section{Implementation Issues}

A bottleneck of Hamiltonian method is the requirement of $D D^{T}-I$ being nonsingular. This problem limits the applications of Hamiltonian method. Fortunately, GHM remains applicable after a small modification process called the equivalent model conversion [1]. The basic idea of equivalent model conversion is to construct a new DS $S^{\prime}(j \omega)=C^{\prime}\left(j \omega E^{\prime}-\right.$ $\left.A^{\prime}\right)^{-1} B^{\prime}+D^{\prime}$ such that $S^{\prime}(j \omega)=S(j \omega)$ with $D^{\prime} D^{\prime T}-I$ being invertible. $S^{\prime}(j \omega)$ can be constructed in different ways. In this paper, we construct $S^{\prime}(j \omega)$ as follows:

$$
\begin{aligned}
& E^{\prime}=\left[\begin{array}{ll}
E & \\
& 0
\end{array}\right], \quad A^{\prime}=\left[\begin{array}{ll}
A & \\
& I
\end{array}\right], \quad B^{\prime}=\left[\begin{array}{c}
B \\
I
\end{array}\right], \\
& C^{\prime}=\left[\begin{array}{ll}
C & -D
\end{array}\right], D^{\prime}=0 .
\end{aligned}
$$

After equivalent model conversion, one can test the passivity of $S(j \omega)$ by performing GHM on $S^{\prime}(j \omega)$. We remark that the equivalent model conversion on standard state-space models yields DS models, which can not be tested by Hamiltonian method but can be tackled by GHM.

The whole passivity test flow can be summarized as follows.

Step 1: Perform Moebius transform to get a "scattering" DS if the original DS is a hybrid one, and then proceed to Step 2. If the original DS is a scattering DS, proceed to Step 2 directly.

Step 2: Check if $E$ and $I-D^{T} D$ are both nonsingular. If yes, convert the DS to a standard state-space model and check system passivity by (3), and then go to Step 5; otherwise (i.e., either $E$ or $I-D^{T} D$ is singular), proceed to Step 3 .

Step 3: Form the matrix pencil in (15), with equivalent model conversion in advance in case $I-D^{T} D$ is singular. Go to Step 4.

Step 4: Compute the generalized eigenvalues of $\left(M_{0}, N_{0}\right)$ in (15). Go to Step 5.

Step 5: Identify the possible nonpassive regions as follows.

Step 5.1: If no crossover points are found, we check $S\left(j \omega_{0}\right)$ at an arbitrarily selected frequency point $\omega_{0}$. If $\left\|S\left(j \omega_{0}\right)\right\|<1$, the DS is passive at any frequency point. Otherwise, the DS is consistently nonpassive .

Step 5.2: If $p$ ascending crossover points $\hat{\omega}_{1}, \hat{\omega}_{2}, \ldots, \hat{\omega}_{p}$ are obtained, we select $p+1$ sampling points $\omega_{k}(k=1,2, \ldots, p+1)$ such that $\omega_{k} \in \ell_{k}$ where $\ell_{1}=\left(0, \hat{\omega}_{1}\right), \ell_{i}=\left(\hat{\omega}_{i-1}, \hat{\omega}_{i}\right)$ for $i=$ $2, \ldots, p$ and $\ell_{p+1}=\left(\hat{\omega}_{p}, \infty\right)$. If $\left\|S\left(j \omega_{k}\right)\right\|<1$, then the DS is passive in the interval $\ell_{k}$, otherwise nonpassive in $\ell_{k}$.

Analogous to the hybrid GHM test, the main computation of the proposed $S$-parameter GHM test is the $O\left(n^{3}\right)$ generalized eigenvalue computation.

\section{EXAMPLES}

We demonstrate the validity and effectiveness of GHM test with some practical examples. The Matlab function "eig" is used as the generalized/standard eigenvalue solver.

\section{A. An $S$-parameter Three-Terminal Filter}

We use the standard state-space model to illustrate the validity of GHM and its connection with traditional Hamiltonian method. The frequency-dependent scattering parameters are measured at 1601 sampling points ranging from $50 \mathrm{MHz}$ to $6 \mathrm{GHz}$. We first build an order-120 standard state-space model $(A, B, C, D)$, which is approximated by vector fitting with 40 common poles. The traditional Hamiltonian method [10] shows that this system contains 9 crossover points. Based on the obtained standard state-space model, we also build a DS 
(a)

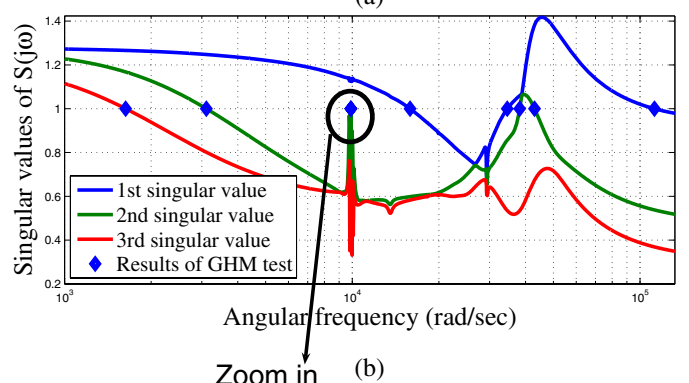

Zoom in (b)

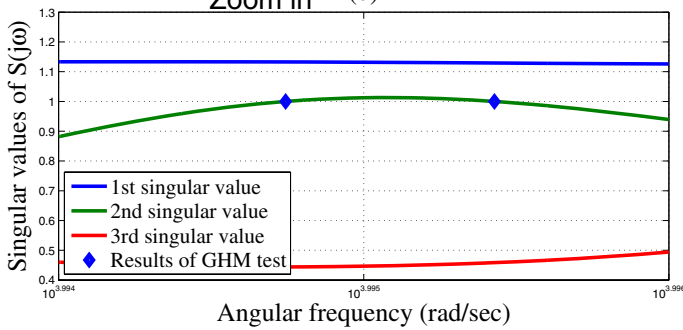

Angular frequency $(\mathrm{rad} / \mathrm{sec})$

Fig. 1. GHM test results for the 3-terminal filter.

TABLE I

EXPERIMENTAL RESULTS OF DIFFERENT PASSIVITY TESTS FOR THE 3-TERMINAL FILTER MODEL.

\begin{tabular}{|c|c|}
\hline $\begin{array}{c}\text { Hamiltonian method: } \\
\text { imaginary results of } \lambda\left(\Pi_{S}\right)\end{array}$ & $\begin{array}{c}\text { GHM: imaginary } \\
\text { results of } \lambda\left(M_{0}, N_{0}\right)\end{array}$ \\
\hline $6.9 \mathrm{e}-10 \pm j 112253$ & $2.17 \mathrm{e}-7 \pm j 112253$ \\
\hline $2.6 \mathrm{e}-10 \pm j 42988.4$ & $2.58 \mathrm{e}-8 \pm j 42988.4$ \\
\hline $1.3 \mathrm{e}-10 \pm j 38173.7$ & $1.06 \mathrm{e}-8 \pm j 38173.7$ \\
\hline $2.2 \mathrm{e}-11 \pm j 34551.3$ & $2.93 \mathrm{e}-8 \pm j 34551.3$ \\
\hline $4.0 \mathrm{e}-12 \pm j 3109.22$ & $2.80 \mathrm{e}-6 \pm j 3109.22$ \\
\hline $2.1 \mathrm{e}-12 \pm j 1631.07$ & $6.02 \mathrm{e}-7 \pm j 1631.07$ \\
\hline $3.0 \mathrm{e}-10 \pm j 15871.8$ & $1.85 \mathrm{e}-8 \pm j 15871.8$ \\
\hline $8.7 \mathrm{e}-11 \pm j 9879.71$ & $5.17 \mathrm{e}-7 \pm j 9879.71$ \\
\hline $8.5 \mathrm{e}-11 \pm j 9895.29$ & $5.20 \mathrm{e}-7 \pm j 9895.29$ \\
\hline
\end{tabular}

model $H_{d}(j \omega)$ described by $\left(E_{d}, A_{d}, B_{d}, C_{d}, D_{d}\right)$, with

$$
\begin{aligned}
& E_{d}=\left[\begin{array}{ll}
I_{120} & \\
& 0
\end{array}\right], A_{d}=\left[\begin{array}{ll}
A & \\
& I_{3}
\end{array}\right] \\
& B_{d}=\left[\begin{array}{l}
B \\
I_{3}
\end{array}\right], C_{d}=\left[\begin{array}{ll}
C & -D
\end{array}\right], D_{d}=0
\end{aligned}
$$

Note that the DS model can also be built directly by DS-format vector fitting. GHM test on $H_{d}(j \omega)$ also reports 9 crossover points. The experimental results of both methods pinpoint the same boundary frequency points, which coincide with the singular value curves of the transfer matrix demonstrated in Fig. 1. We also list the numerical results in Table I. There are some numerical noise in the real parts of GHM and Hamiltonian test results, which can be eliminated by setting a small numerical tolerance [1].

\section{B. An Admittance PEEC Reduced Model}

The original admittance SISO model is an order-480 DS. We get an order-53 reduced model via PRIMA [3], which is non- (a)
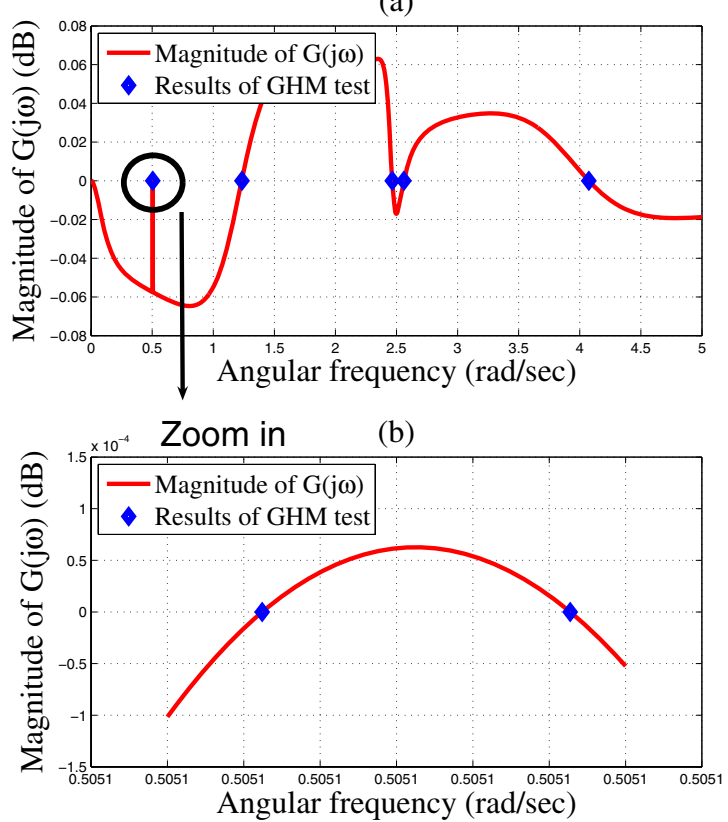

Fig. 2. GHM test results on the Moebius-transformed transfer function of the order-53 admittance reduced model.

TABLE II

GHM TEST RESULTS FOR THE ADMITTANCE REDUCED MODEL (ON THE MOEBIUS-TRANSFORMED SYSTEM).

\begin{tabular}{|c|c|}
\hline $\begin{array}{c}\text { GHM: imaginary } \\
\text { results of } \lambda\left(M_{0}, N_{0}\right)\end{array}$ & $\begin{array}{c}\text { Crossover } \\
\text { points }\end{array}$ \\
\hline $1.309 \mathrm{e}-11 \pm j 0.505080$ & 0.505080 \\
\hline $1.307 \mathrm{e}-11 \pm j 0.505082$ & 0.505082 \\
\hline $1.127 \mathrm{e}-13 \pm j 1.234402$ & 1.234402 \\
\hline $3.650 \mathrm{e}-13 \pm j 2.465012$ & 2.465012 \\
\hline $3.169 \mathrm{e}-13 \pm j 2.560446$ & 2.560446 \\
\hline $7.587 \mathrm{e}-13 \pm j 4.074095$ & 4.074095 \\
\hline
\end{tabular}

passive in the low-frequency band. We construct the system matrices of the corresponding Moebius transformed function $G(s)$, and then test the bounded realness of $G(s)$ by GHM after equivalent model conversion. Computed crossover points are listed in Table II. Fig. 2 shows that the magnitude of the Moebius-transformed transfer function equals unity at the computed crossover points. We also plot the real part of the original admittance transfer function $S(j \omega)$ in Fig. 3, which shows the proposed GHM method can accurately locate the nonpassive regions of hybrid DSs.

\section{CONCLUSION}

We have extended the generalized Hamiltonian method to $S$ parameter DSs. By virtue of the proposed method, the passivity of $S$-parameter and hybrid DSs can be tested with accurate identification of the passive and nonpassive frequency regions. The proposed $S$-parameter GHM method is efficient in testing the validity of reduced circuit models. It is also expected to 

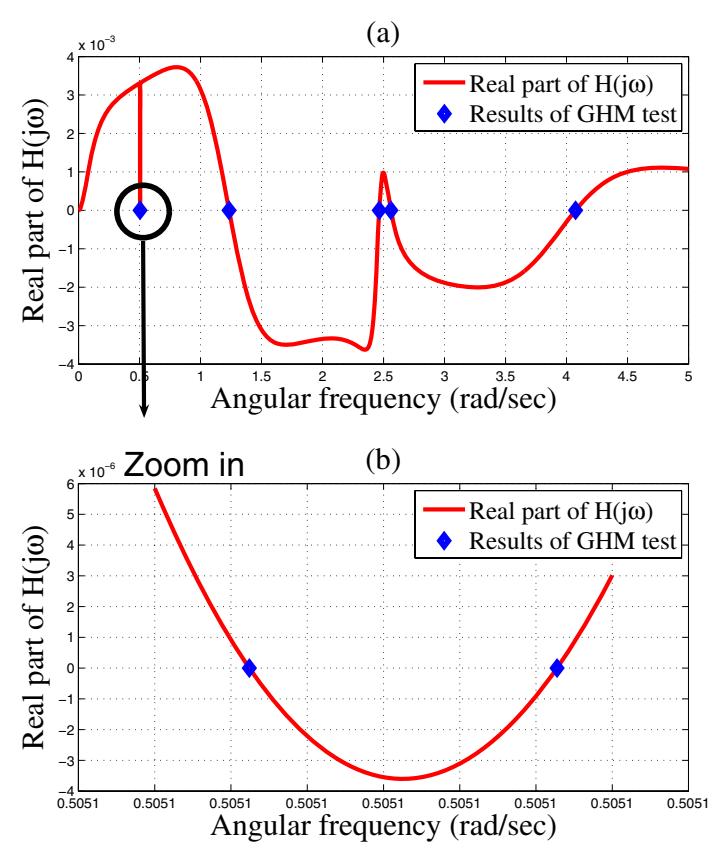

Fig. 3. The real part of the transfer function of the original order-53 admittance DS model.

speed up the passivity compensations of nonpassive DS models generated by macromodeling or MOR techniques.

\section{ACKNOWLEDGMENTS}

This work is supported in part by the Hong Kong Research Grant Council under projects HKU 717407E and 718509E, and the University Research Committee of The University of Hong Kong.

\section{REFERENCES}

[1] Z. Zhang and N. Wong, "GHM: A generalized Hamiltonian method for passivity test of impedance/admittance descriptor systems," in Proc. Intl. Conf. Computer-Aided Design. San Jose, CA, Jan 2009, pp. 767-773.

[2] N. Marques, M. Kamon, L. Silveira, and J. White, "Generating compact, guaranteed passive reduced-order models of 3-D RLC interconnects," IEEE Trans. Advanced Packaging, vol. 27, no. 4, pp. 569-580, Nov 2004.

[3] A. Odabasioglu, M. Celik, and L. Pileggi, "PRIMA: Passive and reducedorder interconnect macromodeling algorithm," IEEE Trans. Comput.Aided Desesign, vol. 17, no. 8, pp. 645-654, Aug 1998.

[4] T. Reis and T. Stykel, "Passivity-preserving balanced truncation for electrical circuits," 2008, technical report, available at http://www.math.tuberlin.de/ stykel/.

[5] N. Marques, M. Kamon, J. White, and L. Silveira, "A mixed nodal-mesh formulation for efficient extraction and passive reduced-order modeling of 3d interconnects," in Proc. Design Automation Conference, 1998, pp. 297-302.

[6] D. Ioan and G. Ciuprina, "Reduced order models of on-chip passive components and interconnects, workbench and test structures," in Model Order Reduction: Theory, Research Aspects and Applications. Springer, Berlin Heidelberg, Aug 2008, pp. 447-467.
[7] H. Wu and A. C. Cangellaris, "Model-order reduction of finite-element approximations of passive electromagnetic devices including lumped electrical-circuit models," IEEE Trans. Microwave Theory and Tech., vol. 52, no. 9, pp. 2305 - 2313, Sep 2004.

[8] T. Stykel, "Gramian based model reduction for descriptor systems," Mathematics of Control, Signals, and Systems, vol. 16, no. 4, pp. 297-319, Mar 2004.

[9] B. Gustavsen and A. Semlyen, "Rational approximation of frequency domain responses by vector fitting," IEEE Trans. Power Delivery, vol. 14, no. 3, pp. 1052-1061, July 1999.

[10] S. Grivet-Talocia, "Passivity enforcement via perturbation of Hamiltonian matrices," IEEE Trans. Circuits Syst. I, vol. 51, no. 9, pp. 1755-1769, Sept 2004.

[11] D. Saraswat, R. Achar, and M. Nakhla, "Fast passivity verification and enforcement via reciprocal systems for interconnects with large order macromodels," IEEE Trans. VLSI Syst., vol. 15, no. 1, pp. 48-59, Jan 2007.

[12] R. W. Freund, "An extension of positive real lemma to descriptor systems," Optimization methods and software, vol. 19, no. 1, pp. 69-87, Feb 2004.

[13] N. Wong and C. K. Chu, "A fast passivity test for stable descriptor systems via skew-Hamiltonian/ Hamiltonian matrix pencil transformations," IEEE Trans. Circuits and Syst. I, vol. 55, no. 2, pp. 635-643, Mar 2008.

[14] P. Benner and D. Chu, "A new test for passivity of descriptor systems," Nov 2005, oberwolfach Reports, Mathematisches Forschungsinstitut Oberwolfach.

[15] Y. Liu and N. Wong, "Fast sweeping methods for checking passivity of descriptor systems," in Proc. Asia Pacific Conf. Circuits Syst., Dec 2008, pp. 566-569.

[16] H. S. Wang, C. F. Yung, and F. R. Chang, "Bounded real lemma and $H_{\infty}$ control for descriptor systems," IEE Proc. Control theory appl., vol. 145, no. 3, pp. 316-322, 1998.

[17] B. Brogliato, R. Lozano, B. Maschke, and O. Egeland, Dissipative Systems Analysis and Control: Theory and Applications, 2nd ed. Springer, 2007. 